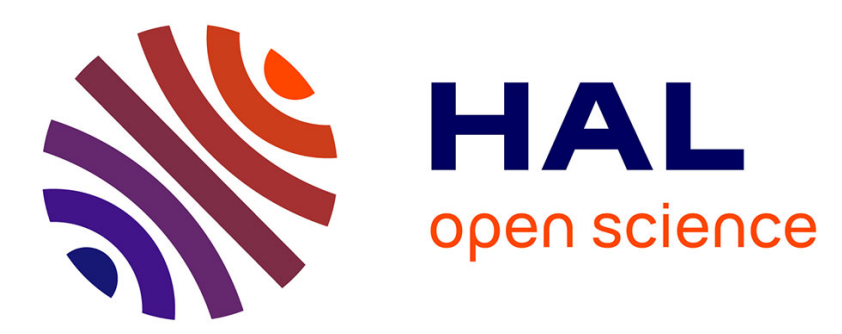

\title{
A new structure for enhanced transmission through a two-dimensional metallic grating
}

D. van Labeke, F.I Baida, Jm Vigoureux

\section{To cite this version:}

D. van Labeke, F.I Baida, Jm Vigoureux. A new structure for enhanced transmission through a two-dimensional metallic grating. Journal of Microscopy, 2004, 213 (2), pp.140-143. 10.1111/j.13652818.2004.01272.x . hal-00097017

\section{HAL Id: hal-00097017 \\ https://hal.science/hal-00097017}

Submitted on 20 Apr 2021

HAL is a multi-disciplinary open access archive for the deposit and dissemination of scientific research documents, whether they are published or not. The documents may come from teaching and research institutions in France or abroad, or from public or private research centers.
L'archive ouverte pluridisciplinaire HAL, est destinée au dépôt et à la diffusion de documents scientifiques de niveau recherche, publiés ou non, émanant des établissements d'enseignement et de recherche français ou étrangers, des laboratoires publics ou privés. 


\title{
A new structure for enhanced transmission through a two-dimensional metallic grating
}

\author{
D. VAN LABEKE, F. I. BAIDA \& J.-M. VIGOUREUX* \\ Laboratoire d'Optique P.M. Duffieux, CNRS UMR 6603, IMFC FR0067, and *Laboratoire de \\ Physique Moléculaire, CNRS UMR 6624, Université de Franche-Comté, Route de Gray, 25030 \\ Besançon Cedex, France
}

Key words. Diffraction gratings, light transmission, metallic thin films,

nanostructured materials.

\section{Summary}

The spectral response of metallic two-dimensional periodic structures in which circular apertures are engraved has been extensively studied. We show that in such devices transmission can be highly enhanced by partially shutting the central parts of each aperture in order to make a periodic array of subwavelength coaxial structures.

\section{Introduction}

Since the publication of T. W. Ebbesen et al. in 1998 (Ebbesen et al., 1998), many experimental and theoretical studies have been carried out in order to determine the physical origin of the enhanced transmission that they obtained. Effects of surface plasmon resonances (Ghaemi et al., 1998; Schröter \& Heitmann, 1998; Porto et al., 1999; Krishnan et al., 2001) or of cavity resonances (Lopez-Rioz et al., 1998; Astilean et al., 2000; Lalanne et al., 2000; Popov et al., 2000) have been proposed to explain the origin of the enhanced transmission. This enhancement of transmitted light is similar to that observed with frequency selective surface (FSS) components in the spectral region from near-infrared to microwave wavelengths ( $\mathrm{Wu} \&$ Lee, 1994; Winnewisser et al., 1999; and references therein).

FSS components are intended for a broad range of applications, such as Fabry-Pérot interferometers, filters, couplers for laser cavity output or simply polarizers. Their spectral responses are directly linked to their geometrical parameters, i.e. their thickness, size, period and in particular their shape. We propose a new structure showing an interesting spectral response in the visible region. Indeed, to increase the transmission of a metal film perforated with subwavelength apertures, it is intuitive to look for a structure in which the apertures have no cut-off frequency in the desired spectral range. This property is verified for the TEM (transverse electro-

Correspondence to: Dr F. I. Baida. E-mail; fbaida@univ-fcomte.fr magnetic) mode in a coaxial waveguide made in a pure metal (Landau \& Lifshitz, 1960). It is well known that a coaxial cable can transmit electromagnetic fields with wavelengths much larger than their radii. Thus, we study the transmission of light by a two-dimensional (2D) periodic structure made of annular apertures instead of a hole grating.

Our first results show that such a geometry rseults in a large increase of transmissivity.

\section{Numerical model and simulations}

The principle of the numerical method employed, i.e. the finite-difference time domain (FDTD) method, can be found in many textbooks (Sadiku, 2000; Taflove \& Hagness, 2000). For calculations, we use a home-made FDTD code. This includes the second-order Mur technique to solve the parasitical reflections at the edges of the grid (Mur, 1981). Metal dispersion is taken into account by using a Drude model to describe the frequency dependence of the permittivity as in Parent et al. (2001). The validity and the accuracy of our code have been checked by reproducing published transmission curves of the 1D metallic grating studied in Astilean et al. (2000).

Our system comprises a periodic array of annular apertures (Fig. 1), the external and internal radii of which are $r_{1}$ and $r_{2}$, respectively. The thickness of the film is $e$ and the period is $p$, in both the $x$ and the $y$ directions. The permittivity of the incident medium, of the transmission medium and of the metal are denoted $\varepsilon_{1}, \varepsilon_{3}$ and $\varepsilon_{2}$, respectively. In this study, both incident and transmission media are vacuum, i.e. $\varepsilon_{1}=\varepsilon_{3}=1$. The refractive index of the in-apertures media is denoted $\varepsilon_{0}$; its value is 1 except where explicitly given. The illumination is a linearly polarized pulse plane wave that hits the metallic film under normal incidence. The pulse is centred at $600 \mathrm{~nm}$ and has a temporal width that permits coverage of the entire visible region of the spectrum.

Figure 2 shows the spectral responses of a 2D array of annular apertures for two different radii of the annular aperture. 
Fig. 1. Schematic of the structure. Annular apertures are made in a metallic film. $r_{1}$ is the external radius and $r_{2}$ the internal radius. $p$ is the period in both the $x$ and the $y$ directions. $\varepsilon_{1}, \varepsilon_{2}$ and $\varepsilon_{3}$ are the incident, metallic and transmission media permittivity. $e$ is the thickness of the metallic film. Illumination is provided by a normally incident plane wave.

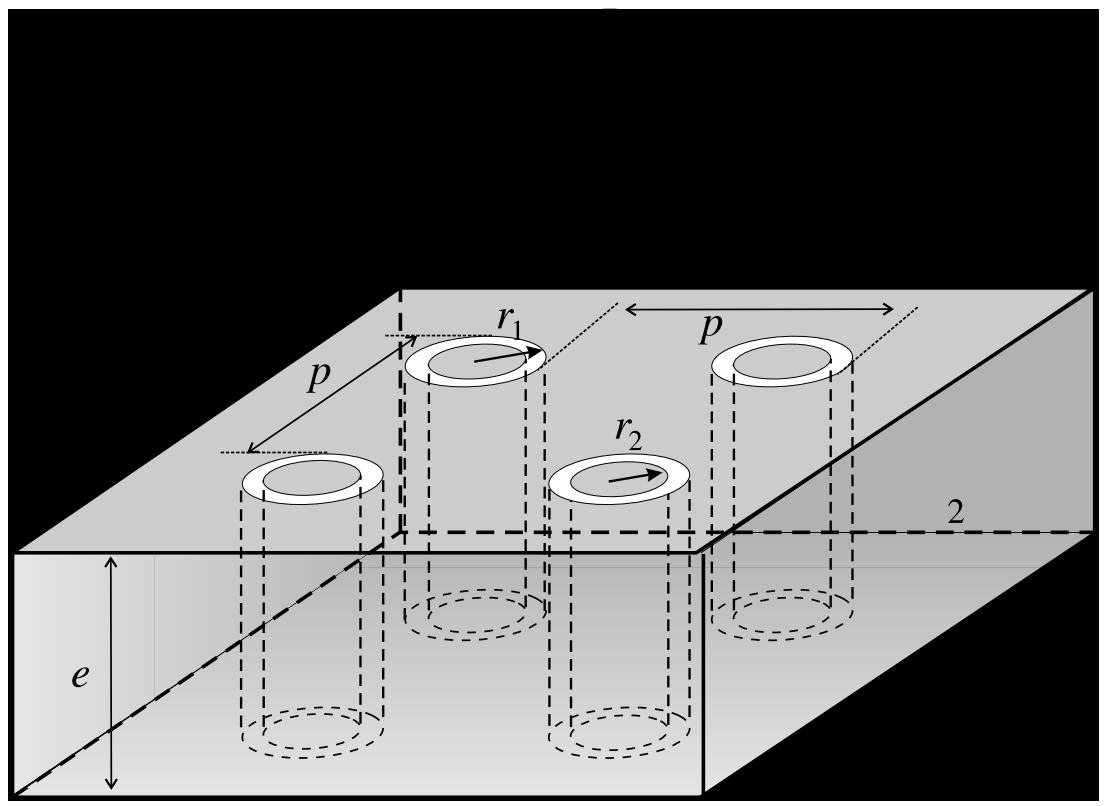

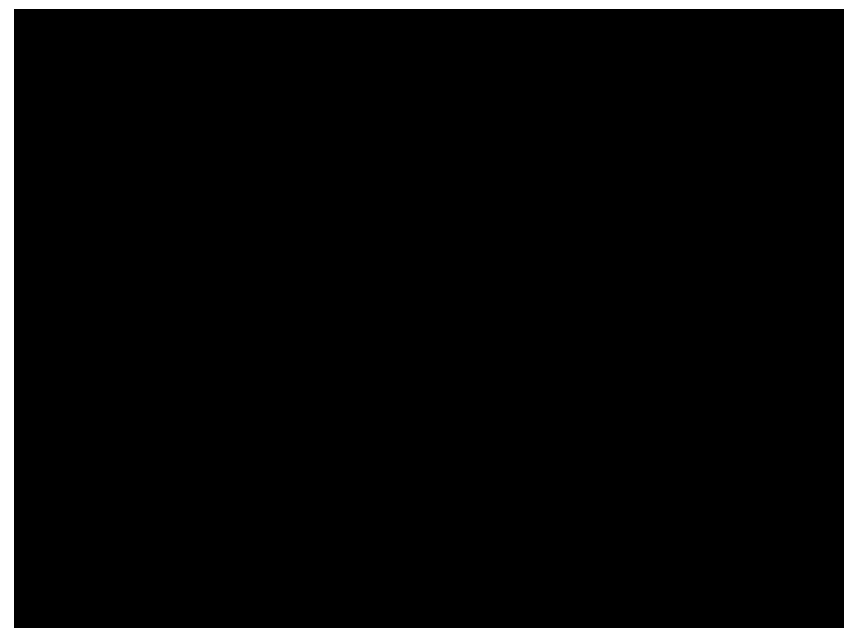

Fig. 2. The spectral responses in the optical range (efficiency of the transmitted zero-order) of a silver 2D array of annular apertures for two different radii of the annular aperture. The parameters of the object are (see Fig. 1): $r_{1}=85 \mathrm{~nm}$ for the solid curve and $r_{1}=75 \mathrm{~nm}$ for the dashed curve. In the two cases, $r_{2}=50 \mathrm{~nm}, p=300 \mathrm{~nm}, \varepsilon_{1}=\varepsilon_{3}=1$ and the thickness is $e=150 \mathrm{~nm}$. Illumination is provided by a x-polarized plane wave at normal incidence.

The spectrum in the visible region is principally composed of two peaks. Their positions and intensities for six metal thicknesses are reported in Table 1 . The position of $\lambda_{2}$ (in the red range of the spectrum) is practically independent of metal thickness, but its intensity decreases when $e$ increases. The other peak $\left(\lambda_{1}\right)$ has a more interesting behaviour: when the metal thickness is increased, it is shifted toward the red region but its intensity remains almost constant. A third peak (a
Table 1. Positions and transmission values of the two peaks appearing in the spectral response of a silver grating for six metal film thicknesses. The parameters of the object are (see Fig. 1): $r_{1}=75 \mathrm{~nm}, r_{2}=50 \mathrm{~nm}$, $p=300 \mathrm{~nm}, \varepsilon_{1}=\varepsilon_{3}=1$. Illumination is provided by an x-polarized plane wave at normal incidence.

\begin{tabular}{lllll}
\hline$e(\mathrm{~nm})$ & $\lambda_{1}(\mathrm{~nm})$ & $E_{0}\left(\lambda_{1}\right)$ & $\lambda_{2}(\mathrm{~nm})$ & $E_{0}\left(\lambda_{2}\right)$ \\
\hline 150 & 514 & 0.6904 & 704 & 0.7883 \\
160 & 525 & 0.7077 & 705 & 0.7783 \\
170 & 535 & 0.7178 & 705 & 0.7656 \\
200 & 563 & 0.7343 & 706 & 0.7253 \\
220 & 579 & 0.7378 & 706 & 0.7002 \\
300 & 625 & 0.7250 & 707 & 0.6127 \\
\hline
\end{tabular}

doublet $\lambda=491 \mathrm{~nm}$ and $\lambda=502 \mathrm{~nm}$ ) appears when $e=300 \mathrm{~nm}$. It is important to note that the red peak is the largest wavelength that can be transmitted by the structure: the transmission efficiency, at normal incidence, vanishes from $\lambda=1 \mu \mathrm{m}$ to $\lambda=2.8 \mu \mathrm{m}$ and probably further.

Figure 3(a) shows the spectral responses (efficiency of the transmitted zero-order) of an array made of circular apertures $\left(r_{2}=0 \mathrm{~nm}\right.$ instead of $r_{2} \neq 0 \mathrm{~nm}$ as in the case of annular apertures) having the same other physical characteristics. It clearly shows that partially shutting each aperture in order to construct small coaxial structures greatly enhances the transmissivity in the visible region (Fig. 3b).

\section{Discussion}

The proposed structure clearly shows a very high transmission in the visible region, which is of interest for several applications 


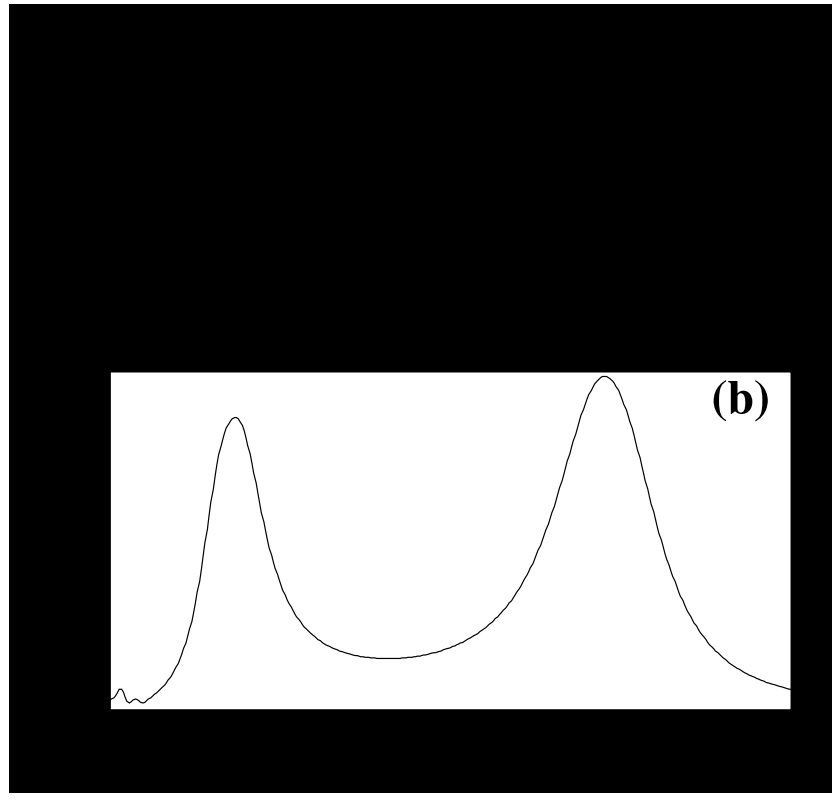

Fig. 3. (a) Zero-order transmission efficiency of a circular aperture grating made in silver film. The geometrical and illumination parameters are defined in Fig. 1: $e=150 \mathrm{~nm}, p=300 \mathrm{~nm}, r_{1}=75 \mathrm{~nm}, r_{2}=0 \mathrm{~nm}$. (b) The zero-order transmission efficiency of the same structure when the central part of each aperture is shut in order to build the corresponding annular aperture grating with $r_{2}=50 \mathrm{~nm}$. Note that the transmitted intensity is greatly enhanced (the scales on the vertical axis are different in (a) and (b), although the free surface of each aperture in (b) is about half that of the apertures in (a)).

such as filters, polarizers or resonators especially in the field of nano-optics. Such a structure with nanometric coaxial apertures could be fabricated with techniques currently available, such as the lift-off process (Li et al., 2000) or focused ion beam (Krishnan et al., 2001; Spajer et al., 2001).

The enhanced transmission of an aperture grating is often interpreted in terms of surface plasmon resonance (see Krishnan et al., 2001, and references therein). Such an interpretation is not possible in the present case: a diffracted wave resulting from the interaction of the incident light with the structure in fact has the same wave-vector tangential component as the surface plasmon one. This leads to the relation (Ghaemi et al., 1998; Krishnan et al., 2001):

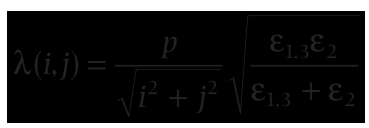

where $i$ and $j$ are the integers that define the diffracted order. In our case ( $p=300 \mathrm{~nm}, \varepsilon_{1}=\varepsilon_{3}=1$ and using silver) the previous equation has no solution for $\lambda>400 \mathrm{~nm}$. So, the enhanced transmission of the coaxial grating cannot be interpreted in terms of excitation of horizontal surface plasmons. Moreover, in Fig. 4 we present two transmission curves corresponding to the same coaxial radii but with a large modification

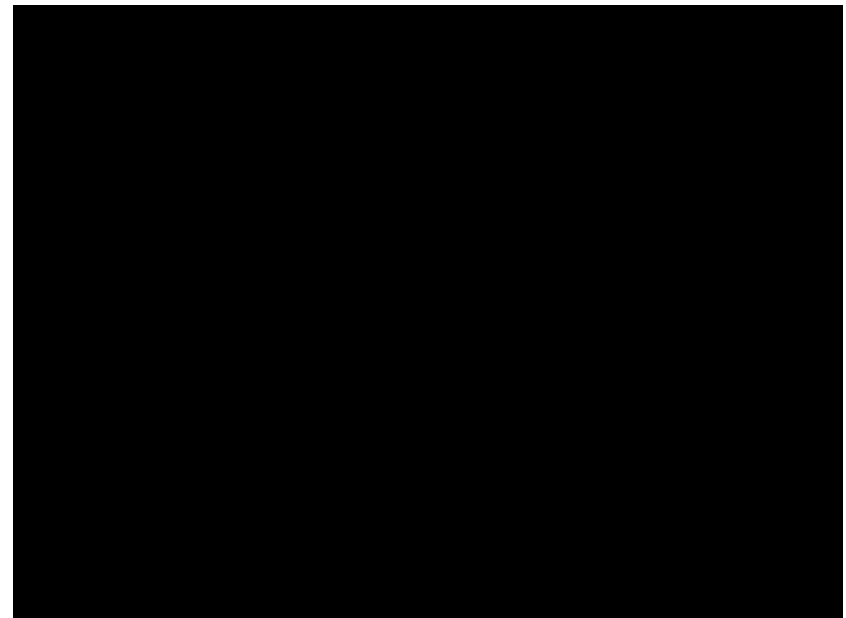

Fig. 4. Zero-order transmission efficiencies of an annular aperture grating made in silver film. The geometrical and illumination parameters are defined in Fig. 1: $e=150 \mathrm{~nm}, r_{1}=75 \mathrm{~nm}, r_{2}=0 \mathrm{~nm}, p=300 \mathrm{~nm}$ for the dashed curve and $p=400 \mathrm{~nm}$ for the solid line.

of the grating period ( $p=300 \mathrm{~nm}$ and $400 \mathrm{~nm}$ ). We notice that the two-peak structure is only slightly modified: only see small shifts of peak position and a decrease of the intensities for larger period.

The results presented in Table 1 suggest that the first peak $\left(\lambda_{1}\right)$ could correspond to a cavity resonance as its position is modified with respect to the metal thickness. When the metal thickness is increased from $150 \mathrm{~nm}$ to $300 \mathrm{~nm}$, the first peak is almost proportionally shifted from $514 \mathrm{~nm}$ to $625 \mathrm{~nm}$.

It can also be noted that the permittivity of the metal also plays a role in the spectral response of the structure: indeed, if silver is replaced by aluminium, the wavelength cut-off is modified. This effect is similar to that observed in a resonant cavity when the phase of the reflection coefficient is modified according to the permittivity of the metallic mirrors. For aluminium, the two-peak structure is maintained but is shifted toward the blue region. The $\lambda_{2}$ peak is shifted by $130 \mathrm{~nm}$ in comparison with that for silver.

\section{Conclusion}

We have numerically studied the spectral responses of a subwavelength annular aperture array exhibiting an very high transmission. We demonstrate the presence of a guided mode through the apertures but we have not been able to determine the nature of this mode (is it really a TEM mode?) nor how it is created. Many other numerical simulations will be necessary to determine the nature of this phenomenon fully. Comparisons with others method, such as the modal method (Popov et al., 2000, and references therein) are in progress. Preliminary results reproduce our transmission curves and seem to confirm the interpretation in terms of cavity resonance. In this way, we have performed calculations with a single annular 
aperture in a 300-nm-thick Ag film. We again obtain the same double peak structure for the spectral response. This result confirms that the enhanced transmission is directly linked to the excitation of surface plasmons by a grating diffraction effect. The results of Fig. 4 are in agreement with this interpretation: a large modification of the grating period leads only to small changes of the two-peak structure.

Finally, independently of the interpretation of enhanced transmission, it is important to emphasize that the proposed structure could have interesting applications. Calculation of the near-field above the grating and of the field inside the cavities is too detailed to be included in this short paper. We note only that the field amplitude in the cavities, and in the nearfield, is very large and can reach 600 times the incident amplitude. The sensitvity of the peak position with respect to the cavity index, in addition to the existence of a very high electric field amplitude, can lead to interesting phenomena if a non-linear material is placed in the apertures.

\section{Acknowledgements}

This paper was first presented at the NFO-7 conference held in Rochester, NY, 11-15 August 2002.

\section{References}

Astilean, S., Lalanne, Ph. \& Palamaru, M. (2000) Light transmission through metallic channels much smaller than the wavelength. Opt. Commun. 175, 265-273.

Ebbesen, T.W., Lezec, H.J., Ghaemi, H.F., Thio, T. \& Wolff, P.A. (1998) Extraordinary optical transmission through sub-wavelength hole arrays. Nature, 391, 667-669.

Ghaemi, H.F., Thio, T., Grupp, D.E., Ebessen, T.W. \& Lezec, H.J. (1998) Surface plasmons enhance optical transmission through sub-wavelength holes. Phys. Rev. B, 58, 6779-6782.

Krishnan, A., Thio, T., Kim, T.J., Lezec, H.J., Ebbesen, T.W., Wolff, P.A., Pendry, J., Martin-Moreno, L. \& Garcia-Vidal, F.J. (2001) Evanescently coupled resonance in surface plasmon enhanced transmission. Opt. Commun. 200, 1-7.

Lalanne, Ph., Hugonin, J.P., Astilean, S., Palamaru, M. \& Möller, K.D. (2000) One-mode model and airy-like formulae for one-dimensional metallic gratings. J. Opt. A: Pure Appl. Opt. 2, 48-51.

Landau, L.D. \& Lifshitz, E.M. (1960) Electrodynamics of Continuous Media. Pergamon Press, Oxford, U.K.

Li, M., Wang, J., Zhuang, L. \& Chou, S.Y. (2000) Fabrication of circular optical structures with a $20 \mathrm{~nm}$ minimum feature size using nanoimprint lithography. Appl. Phys. Lett. 76, 673-675.

Lopez-Rioz, T., Mendoza, D., Garcia-Vidal, F.J., Sanchez-Dehesa, J. \& Pannetier, B. (1998) Surface shape resonances in lamellar metallic gratings. Phys. Rev. Lett. 81, 665-668.

Mur, G. (1981) Absorbing boundary conditions for the finite-difference approximation of the time-domain electromagnetic field equations. IEEE Trans. Electromagnetic Compatibility, 23, 377-382.

Parent, G., Van Labeke, D. \& Baida, F.I. (2001) Theoretical study of transient phenomena in near-field optics. J. Microsc. 202, 296-306.

Popov, E., Nevière, M., Enoch, S. \& Reinisch, R. (2000) Theory of light transmission through subwavelength periodic hole arrays. Phys. Rev. B, 62, 16100-16108.

Porto, J.A., Garcia-Vidal, F.T. \& Pendry, J.B. (1999) Transmission resonances on metallic gratings with narrow slits. Phys. Rev. Lett. 83 , 2845-2848.

Sadiku, M.N.O. (2000) Numerical Techniques in Elelectrodynamics, 2nd edn. CRC Press, Boca Raton, FL, U.S.A.

Schröter, U. \& Heitmann, D. (1998) Surface-plasmon enhanced transmission through metallic gratings. Phys. Rev. B, 58, 15419-15421.

Spajer, M., Parent, G., Bainier, C. \& Charraut, D. (2001) Shaping the reflection near-field optical probe: finite domain time difference modelling and fabrication using a focused ion beam. J. Microsc. 202, 45-49.

Taflove, A. \& Hagness, S.C. (2000) Computational Electrodynamics, the Finite-Difference Time-Domain Method. Artech House, Norwood, MA, U.S.A.

Winnewisser, C., Lewen, F., Weinzierl, J. \& Helm, H. (1999) Transmission features of frequency-selective components in the far field determined by terahertz time-domain spectroscopy. Appl. Opt. 38, 3961-3967.

Wu, T.-K. \& Lee, S.-W. (1994) Multiple frequency selective surface with multiring patch elements. IEEE Trans. Antennas Propagation, 42, 1484-1490. 